$\stackrel{a}{\longrightarrow} \longrightarrow$

$b$

$c$
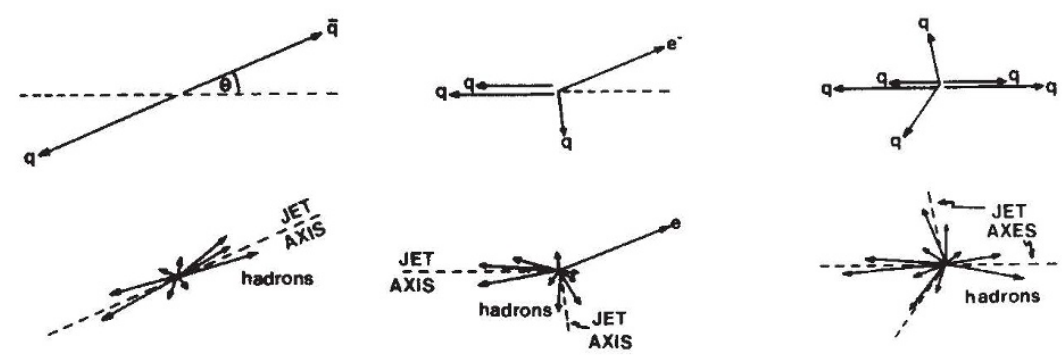

Fig. $1 a-c$; Three classes of high energy particle collisions producing jets of hadrons. The initial state, the inferred primary products and the final state hadrons are shown as momentum vectors.

interpretation is that a pair of constituents (one from each proton) has scattered through a large angle.

From these three types of collision process studied at three quite different types of machine $\left(\mathrm{e}^{+} \mathrm{e}^{-}\right.$colliders at SPEAR and DESY, large accelerators at FNAL and the CERN SPS, and the CERN proton Intersecting Storage Rings (ISR) a unified programme of research has now emerged. The first process (Fig. 1a) is the ideal way to study the mechanism by which quarks evolve into hadrons, the second $(1 b)$ can measure the distribution of quarks inside a proton and the third $(1 c)$ can study the quark-quark scattering process. This threefold attack on the study of the strong interaction was the subject of a Jet Symposium held last year at the Neils Bohr Institute in Copenhagen (Physica Scripta 19, (2) 1979).

Quite how the emerging quarks turn themselves into hadrons is still something of a mystery. Our present understanding of the strong forces between quarks is based on quantum chromodynamics theory (QCD). In this theory the quark-quark force increases as their separation increases, unlike electromagnetic forces where the reverse is true. It may then take an infinite amount of energy to separate a pair of quarks to macroscopic distances. As quarks separate, the energy contained in the increasingly strong field builds up until it is sufficient to "polarise the vacuum', resulting in a newly created quark-antiquark pair. This process repeats itself until most of their kinetic energy is spent, and the created quarks and antiquarks arrange themselves into hadrons. Unfortunately it is not yet possible to calculate the details of this complicated process. It is still an open question whether free isolated quarks can be produced in sufficiently high energy collisions-it is conjectured, but not yet proved, that they are permanently confined. In that case we may now be exploring the last rung of the ladder down from atoms through nuclei and elementary particles to their most fundamental constituents.

\section{Arabic science at Aleppo}

from Subir K. Banerjee

For historians of Arabic science an important meeting* took place in Aleppo, recently. The President of this young university Ahmad Y. Hassan, himself a noted historian of technology, has single-handedly provided the impetus for the creation of the world's first major centre devoted to a comprehensive study of all the Arabic sciences, the Institute for the History of Arabic Science at Aleppo University. The institute is also responsible for the publication of the Journal for the History of Arabic Science, the first journal of its kind. The term Arabic Science is used in preference to Islamic Science, perhaps to emphasise the idea that we are dealing here with the history of science (and technology) written in the Arabic language which was the language of rational inquiry in the Occident during the period AD 800-1400.

Why should anyone study Arabic science? Before we in the modern West mumble something about its impact on the European Renaissance it is worth recalling what E. S. Kennedy, one of the doyens of studies in Arabic mathematics and astronomy has said, "There need be no laboring of the conceited notion that the providential function of the Arabs was to preserve Greek science against the time when Western Man should awake from his slumbers of the Dark Ages". Taking this view one step further I would like to say that while intrinsically laudable, it is an extremely parochial and ethnocentric view to emphasise in the study of Arabic science only the role of transmission to the Latin West at the cost of minimising the grandeur and

* The Second International Symvosium for the at Aleppo, Syria.

Subir K. Banerjee is Professor of Geophysics and Adjunct Professor, Program in Middle Eastern and Islamic Studies, University of Minnesota, Minneapolis. History of Arabic Science was held on 5-12 April breadth of Arabic science as science per se. It is a sobering thought that perhaps only $10 \%$ of the total Arabic scientific oeuvre is known to have been translated into Hebrew and Latin. The growth, maturity and decline of Arabic science should be studied primarily as the flowering of human mind during a certain era and for the lessons it can offer to all mankind.

The symposium was successful in driving home this broader viewpoint of Arabic science. One interesting contribution in the sessions on the 'Transmission of Arabic science to the Latin West' was Marie Therésé d'Alverny's discovery of the important influence of Arabic cosmology in the 12 th century Dialogus of Peter Alphonsi. A highlight was A. I. Sabra's talk in the general session on the place of science and medicine in medieval Islamic civilisation. Over the past few years in private conversations and in short articles Sabra has indicated that he is struggling with the problem of decline of Arabic science and at the symposium he was able to state clearly his new approach to the discussion of this problem: he is concentrating on the careers of the individual scientists of this age, their training, flourishing and activity later in life. In the topical sessions I attended it was most exciting to hear of G. Saliba's identification of the Damascene astronomer al-'Urdi (13th century) as the original author of the planetary model which constituted an alternative to the Ptolemaic model and that 'Urdi's model appears to have been adopted by the more well-known Qutb ad-Din ash-Shirazi. Other interesting new information selected at random is that the Arabs had a magnetic compass for scientific use as early as 13 th century and that a 14 th century Aleppan astronomer, as-Sarraj, had invented an exceedingly complex universal astrolabe which, additionally, had the equivalent of a slide rule on its back for complex calculations in spherical astronomy, the trisection of an arbitrary angle and the nature of the lunar surface.

\section{Timing time}

from $P . C . W$. Davies

IT has been predicted for 60 years that time runs faster in space than on Earth. Now the prediction has been verified by a direct experiment in which a highprecision clock-an atomic hydrogen maser-was flown into space on board a spacecraft. In a recent issue of General Relativity and Gravitation, two Harvard astrophysicists, R. F. C. Vessot and M. W. Levine, report the preliminary results of the experiment, 